Article

\title{
Theoretical Study of the One Self-Regulating Gene in the Modified Wagner Model
}

\author{
Christophe Guyeux ${ }^{1, *}$, Jean-François Couchot ${ }^{1}$, Arnaud Le Rouzic ${ }^{2}$, Jacques M. Bahi ${ }^{1}$ \\ and Luigi Marangio ${ }^{1}$ \\ 1 Femto-ST Institute, UMR 6174 CNRS, University of Bourgogne Franche-Comté, 90000 Belfort, France; \\ Jean-Francois.Couchot@univ-fcomte.fr (J.-F.C.); jacques.bahi@univ-fcomte.fr (J.M.B.); \\ luigi.marangio@univ-fcomte.fr (L.M.) \\ 2 EGCE, CNRS-IRD-Université Paris-Saclay, 91198 Gif-sur-Yvette, France; Arnaud.Le-Rouzic@legs.cnrs-gif.fr \\ * Correspondence: christophe.guyeux@univ-fcomte.fr; Tel.: +33-038-458-7722
}

Received: 31 January 2018; Accepted: 31 March 2018; Published: 9 April 2018

check for updates

\begin{abstract}
Predicting how a genetic change affects a given character is a major challenge in biology, and being able to tackle this problem relies on our ability to develop realistic models of gene networks. However, such models are rarely tractable mathematically. In this paper, we propose a mathematical analysis of the sigmoid variant of the Wagner gene-network model. By considering the simplest case, that is, one unique self-regulating gene, we show that numerical simulations are not the only tool available to study such models: theoretical studies can be done too, by mathematical analysis of discrete dynamical systems. It is first shown that the particular sigmoid function can be theoretically investigated. Secondly, we provide an illustration of how to apply such investigations in the case of the dynamical system representing the one self-regulating gene. In this context, we focused on the composite function $f_{a}(m \cdot x)$ where $f_{a}$ is the parametric sigmoid function and $m$ is a scalar not in $\{0,1\}$ and we have proven that the number of fixed-point can be deduced theoretically, according to the values of $a$ and $m$.
\end{abstract}

Keywords: gene-network model; Wagner model; discrete dynamical systems

\section{Introduction}

Predicting the effect of a genetic change (differences in the DNA molecule) on a character of interest (which can be related to, e.g., human health, plant and animal production, or evolutionary differences between species) remains a major challenge in biology [1]. This is mainly due to the fact that cell physiology is heavily regulated by complex gene networks, which are able to compensate various genetic defects or environmental disturbances. Understanding how DNA variation translates to observable (phenotypic) variation, due for instance to a modification of the conformation of proteins [2] or DNA [3] a is of obvious importance, although very complex. Being able to tackle this complexity relies, among other things, on our ability to develop realistic models of such gene networks.

There exist several kinds of biological interactions that can be modeled as gene networks (e.g., signal transduction, metabolism, or transcription regulation). The literature proposes several modeling frameworks for each of them based on various biological hypotheses and different time scales [4-7]. More generally, [8] provides a complete review of biological systems inspired by network science. An interesting subset of gene network models does not aim at predicting the behavior of a specific group of identified genes in an organism, but are rather used as a general abstraction of a gene network, in order to study their evolutionary properties in individual-based simulations $[9,10]$. Although naive in terms of biological hypotheses, these models are particularly important because they could contribute to unifying systems biology and evolutionary genetics. 
The most popular framework in this field is the so-called "Wagner Model" (after $[9,11]$ ). This model is an abstraction of the interactions between transcription factors (genes that regulate the expression of other genes). The structure of the $n$-gene network is encoded into a $n \times n$ matrix ( $W$ in the original model, thereafter noted $M$ ), which is constant for an organism, and the status of the gene network (the expression of the $n$ genes of the network) is encoded into a vector $S$ of size $n$. The dynamics of the gene network over a finite number of discrete time steps is modeled as a system of $n$ linear difference equations, often written as $S_{t+1}=F\left(M S_{t}\right)$, where $F(x)=\left[f\left(x_{1}\right), f\left(x_{2}\right), \ldots, f\left(x_{n}\right)\right]$, in which $f$ is a scaling function. The $n^{2}$ elements of matrix $M$ are real numbers representing the influence of gene $j$ on gene $i\left(M_{i j}<0\right.$ for repression, $M_{i j}>0$ for up-regulation, and $M_{i j}=0$ for the absence of interaction). Self regulation is possible (and realistic), i.e., $S_{i i}$ can be different from 0 . The purpose of the scaling function $f$ is to ensure that gene expressions $S$ remain in their domain of definition. The gene network starts from an initial vector of gene expressions $S_{0}$, generally set at an arbitrary level.

In the original setting from [11], gene expressions were discrete and could take only two values, -1 for no expression and 1 for an expressed gene, $f(x)$ was thus a step function $(f(x<0)=-1 ; f(0)=0 ; f(x>0)=1)$. Alternative parameterization include e.g., scaling between 0 and 1 [12]. An overview of the diversity of similar settings is provided in [13]. Recent implementations of the model consider continuous gene expressions, and the step function was turned into a sigmoid, scaling gene expressions between -1 and $1\left(f(x)=2 /\left(1+e^{-x}\right)-1\right.$, see [14,15]). For more realism, the sigmoid function can also be further modified to ensure that genes are only weakly expressed in absence of regulators, by considering that $f(0)=a<1 / 2$, as in [16], and which is the model studied below.

The main purpose of such models is to ensure that any combination $M, S_{0}$ can be solved computationally (e.g., as the state of the network $S_{T}$ after $T$ time-steps) within a predictable (most of the time, constant) amount of time. This is of major importance in individual-based computer simulations or other numerical studies in which the network structure $M$ can mutate and evolve over time. The lack of mathematical tractability remains, however, problematic, as it makes it difficult to compare simulation results with classical predictions from population and quantitative genetics models [17,18].

This is why, in this article, a mathematical analysis of the sigmoid variant of the Wagner gene-network model is presented, focusing on the simplest case, that is, one unique self-regulating gene. It is demonstrated that, in addition to classical numerical simulations, this model can be theoretically studied too, by the means of mathematical analysis of discrete dynamical systems. It is first shown that the particular sigmoid function, usually studied within this model, can be theoretically investigated, and such investigations are secondly partially applied to the dynamical system representing the one self-regulating gene. Finally, some ways to extend the analysis to the multiple gene case are sketched.

The remainder of this article is structured as follows. In the next section, the parameterized sigmoid function is deeply studied by the means of mathematical analysis. Effects of parameter changes on its shape are discussed too, and a systematic investigation of its fixed points is finally provided. Section 3, for its part, focuses on the discrete dynamical system of the modified Wagner model. The one self-regulating gene model is next partially studied, in a particular situation and then in its most general formulation. This article ends by a conclusion section, in which the contribution is summarized and intended future work is outlined.

\section{Studying the Sigmoid Function}

We first provide some rationales about the sigmoid function usually considered in the variant of the Wagner gene-network model studied here [16]. They will be used in the next section, when studying the one self-regulating gene system. 


\subsection{Introducing the Considered Sigmoid}

Let us consider $a \in] 0,1[$ and the particular sigmoid function defined by:

$$
\forall x \in \mathbb{R}, f_{a}(x)=\frac{1}{1+\lambda \mathrm{e}^{-\mu x}}
$$

with $\lambda=\frac{1-a}{a}>0$ and $\mu=\frac{1}{a(1-a)}=\frac{1}{a}+\frac{1}{1-a}>1$.

This function, and its parameters, have been chosen in order to have:

- a continuous increasing function,

- the limit of $f_{a}$ as $x$ approaches negative infinity is 0 ,

- the limit of $f_{a}$ as $x$ approaches infinity is 1 ,

- $f_{a}(0)=a$ and $f_{a}^{\prime}(0)=1$,

which corresponds to the curve depicted in Figure 1.

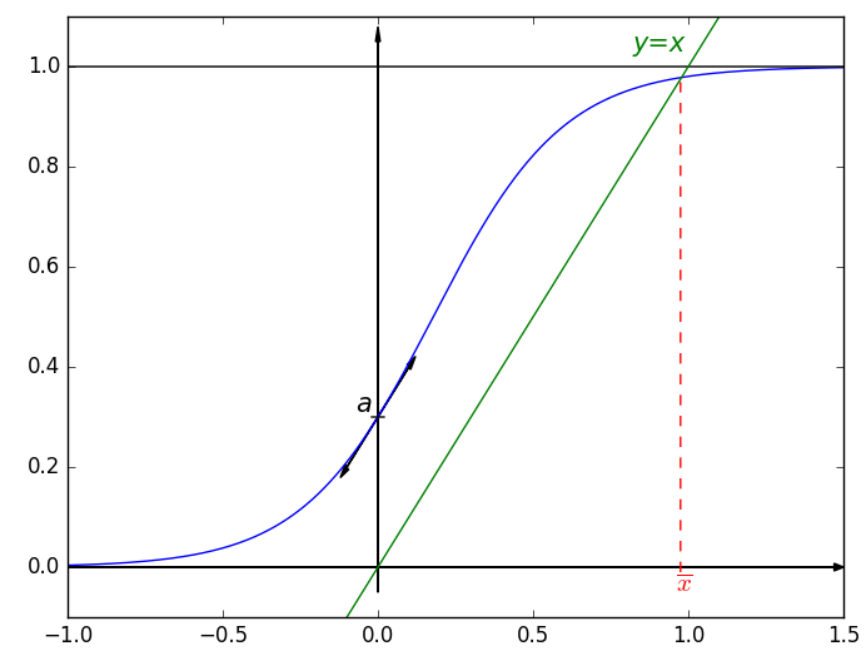

Figure 1. Graph of the sigmoid function $f_{a}(x)=\frac{1}{1+\lambda \mathrm{e}^{-\mu x}}$ with $\lambda=\frac{1-a}{a}>0, \mu=\frac{1}{a(1-a)}$ and $a \in] 0,1[$.

$f_{a}$ is a smooth function, with

$$
\forall x \in \mathbb{R}, f_{a}^{\prime}(x)=\frac{\lambda \mu \mathrm{e}^{-\mu x}}{\left(1+\lambda \mathrm{e}^{-\mu x}\right)^{2}}>0 .
$$

We can thus verify that $f_{a}$ is strictly increasing. Additionally, we can reformulate this derivative, to relate it to the logistic map:

$$
f_{a}^{\prime}(x)=\mu f_{a}(x)\left(1-f_{a}(x)\right) .
$$

\subsection{About $\lambda$ and $\mu$ Parameters}

Let us now investigate the two parameters inside $f_{a}$ that both depend on $a . \lambda(a)=\frac{1}{a}-1$ has a curve depicted in Figure 2a. $\mu(a)=\frac{1}{a}+\frac{1}{1-a}$, for its part, has a derivative equal to:

$$
\mu^{\prime}(a)=\frac{2 a-1}{a^{2}(1-a)^{2}}
$$

whose variation table is as described in Figure 3. 


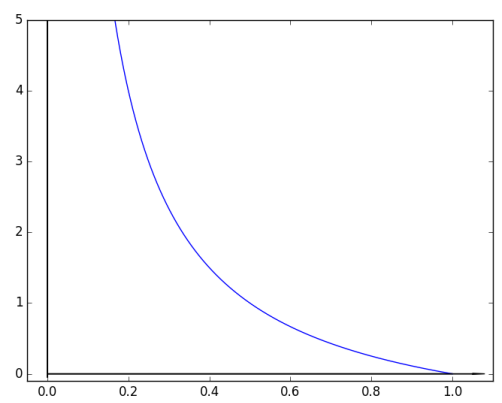

(a) $\lambda(a)=\frac{1-a}{a}>0$

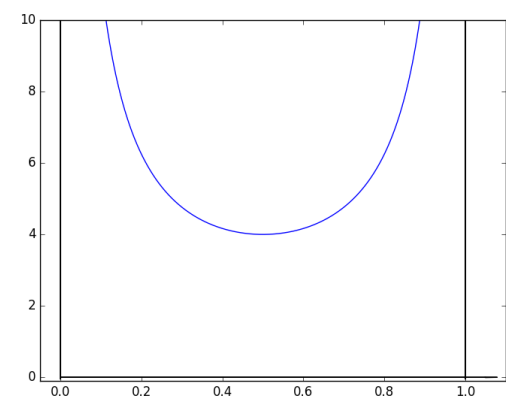

(b) $\mu(a)=\frac{1}{a(1-a)}$

Figure 2. Parameter dependence against $a \in] 0,1[$.

\begin{tabular}{|c|c|c|c|c|c|}
\hline$a$ & 0 & & $\frac{1}{2}$ & & 1 \\
\hline$\mu^{\prime}(a)$ & & - & $\dot{\theta}$ & + & \\
\hline $\mathcal{C}_{\mu}$ & $+\infty$ & & & & $+\infty$ \\
\hline
\end{tabular}

Figure 3. Variation table of $\mu$.

Let us thus remark that $\lambda \mu=\frac{1}{a^{2}}>1$, next that $\left.\frac{\lambda}{\mu}=(1-a)^{2} \in\right] 0,1[$ and finally that $h$ is always greater or equal than 4

\subsection{Fixed Point of $f_{a}$}

One of the most important elements to study, when investigating the Wagner model, is the existence and meaning of fixed points. We will show that the fixed points of the one self-regulating gene model are related to those of the modified sigmoid function $f_{a}$, which are studied hereafter.

We can first remark that, as $\forall a \in[0,1], f_{a}$ is continuous and such that $f_{a}([0,1]) \subset f_{a}([0,1])$, it is thus a function mapping a compact convex set to itself. Due to Brouwer's fixed-point theorem, $f_{a}$ has at least one fixed point in $[0,1]$. Let us prove that,

Theorem 1. $\forall a \in[0,1], f_{a}$ has one unique fixed point in $\mathbb{R}$, which is within $[0,1]$.

Proof. Let us first remark that, if $f_{a}$ has a fixed point $\bar{x}$, then it satisfies:

$$
\bar{x}=\frac{1}{1+\lambda \mathrm{e}^{-\mu \bar{x}}} .
$$

Moreover, as $f_{a}(\bar{x})=\bar{x}$ on the one hand, and $\left.f_{a}(\mathbb{R})=\right] 0,1[$ on the other hand, we necessarily have:

$$
\bar{x} \in] 0,1[\text {. }
$$


Additionally, $0<\bar{x}$ and $f_{a}$ is strictly increasing, so $f_{a}(0)<f_{a}(\bar{x})$, which leads to $a<\bar{x}$. To sum up, if $f_{a}$ has a fixed point $\bar{x}$, then this latter satisfies:

$$
0<a<\bar{x}<1
$$

Let us consider a fixed point $x$ for $f_{a}$. So we have:

$$
f_{a}(x)=x \Longleftrightarrow \frac{1}{1+\lambda \mathrm{e}^{-\mu x}}-x=0 \Longleftrightarrow h(x)=\frac{1}{1+\lambda \mathrm{e}^{-\mu x}}\left(1-x-\lambda x \mathrm{e}^{-\mu x}\right)=0
$$

Let us define $H(x)=1-x-\lambda x \mathrm{e}^{-\mu x}$, so $x$ is a fixed point for $f_{a}$ if and only if it is a zero of $H$ : $H(x)=0$. Let us study the variations of $H$ function.

$$
\begin{aligned}
H^{\prime}(x) & =-1-\lambda \mathrm{e}^{-\mu x}+\lambda \mu x \mathrm{e}^{-\mu x}=\lambda \mathrm{e}^{-\mu x}(\mu x-1)-1 \\
H^{\prime \prime}(x) & =-\lambda \mu \mathrm{e}^{-\mu x}(\mu x-1) \lambda \mu \mathrm{e}^{-\mu x} \\
& =\lambda \mu \mathrm{e}^{-\mu x}(2-\mu x) \\
& >0 \Longleftrightarrow x<\frac{2}{\mu}
\end{aligned}
$$

which leads to the variations depicted in Figure 4.

\begin{tabular}{|c|cccc|}
\hline$x$ & $-\infty$ & $\frac{2}{\mu}$ & $+\infty$ \\
\hline$H^{\prime \prime}(x)$ & & + & $\vdots$ & $\vdots$ \\
$\vdots$ \\
$\mathcal{C}_{H^{\prime}}$ & & $+\infty$ & $\lambda \mathrm{e}^{-2}-1$ & \\
& & & \\
\hline
\end{tabular}

Figure 4. Variations for $H^{\prime}$.

Depending on the sign of $\lambda \mathrm{e}^{-2}-1, H^{\prime}$ can be either negative on the whole $\mathbb{R}$ set, or positive on a bounded interval containing $\frac{2}{\mu}$. More precisely,

$$
\left.\lambda \mathrm{e}^{-2}-1 \geqslant 0 \Longleftrightarrow \lambda \geqslant \mathrm{e}^{2} \Longleftrightarrow \frac{1}{a}-1 \geqslant \mathrm{e}^{2} \Longleftrightarrow a \leqslant \frac{1}{\mathrm{e}^{2}+1} \in\right] 0,1[.
$$

- If $a \geqslant \frac{1}{\mathrm{e}^{2}+1}$, then $H^{\prime} \leqslant 0$ on $\mathbb{R}$, and we have $\lim _{x \rightarrow-\infty} H(x)=\lim _{x \rightarrow-\infty} 1-x-\lambda x \mathrm{e}^{-\mu x}=+\infty$ and $\lim _{x \rightarrow+\infty} H(x)=-\infty$, which leads to the variations depicted in Figure 5.

Consequently, if $a \geqslant \frac{1}{\mathrm{e}^{2}+1}$, then $H(x)=0$ has one unique solution, i.e., $f_{a}$ has one unique fixed point. - If $a \leqslant \frac{1}{\mathrm{e}^{2}+1}$, then there exist two real numbers $x_{1}$ and $x_{2}$ such that the variation table of Figure 6 is satisfied for $H$.

- $H\left(\frac{2}{\mu}\right)=\left(1-\frac{2}{\mu}-\frac{2 \lambda}{\mu} \mathrm{e}^{-2}\right)$. As $\mu \geqslant 4$, we deduce that $\frac{2}{\mu} \leqslant \frac{1}{2}$. Additionally, $\mathrm{e}^{-2}<\frac{1}{4}$ and $\left.\frac{\lambda}{\mu}=(1-a)^{2} \in\right] 0,1\left[\right.$, then $\frac{2 \lambda}{\mu} \mathrm{e}^{-2}<\frac{1}{2}$. As a conclusion, $H\left(\frac{2}{\mu}\right)>0$. 
As $H\left(\frac{2}{\mu}\right)>0, H$ is increasing on $\left[\frac{2}{\mu}, x_{2}\right], H$ decreases on $\left[x_{2},+\infty[\right.$, and the limit of $H$ of $x$ as $x$ approaches $+\infty$ equals $-\infty$, we can deduce that $H(x)=0$ has an unique solution on $\left[\frac{2}{\mu}, \infty\left[\right.\right.$, on a point $\bar{x}>x_{2}$. In particular, $f_{a}$ has one unique fixed point in this interval.

- We show that $H\left(x_{1}\right)>0$, and so $f_{a}$ has no fixed point on $\left.]-\infty, \frac{2}{\mu}\right]$.

$$
\begin{aligned}
H^{\prime}\left(x_{1}\right)= & 0 \text {, then } \lambda \mathrm{e}^{-\mu x_{1}}\left(\mu x_{1}-1\right)-1=0 \Longleftrightarrow \lambda \mathrm{e}^{-\mu x_{1}}=\frac{1}{\mu x_{1}-1} \text {. So: } \\
H\left(x_{1}\right) & =-\left(\lambda x_{1} \mathrm{e}^{-\mu x_{1}}+x_{1}-1\right) \\
& =-\left[\frac{x_{1}}{\mu x_{1}-1}+x_{1}-1\right]=\frac{-\left(\mu x_{1}^{2}-\mu x_{1}+1\right)}{\left(\mu x_{1}-1\right)} .
\end{aligned}
$$

Furthermore, $H^{\prime}\left(\frac{1}{\mu}\right)=\lambda \mathrm{e}^{-1}(1-1)-1<0$, and $H^{\prime}$ is increasing on $\left.]-\infty, \frac{2}{\mu}\right]$ (as $H^{\prime \prime}>0$ on this interval). As $H^{\prime}\left(x_{1}\right)=0$, we can deduce that $x_{1}>\frac{1}{\mu}$. Consequently, $\mu x_{1}-1>0$. Let $j(x)=\mu x^{2}-\mu x+1$. Since $1+\lambda \mathrm{e}^{-\mu x_{1}}>0$, thus $H\left(x_{1}\right)$ is negative if and only if $j\left(x_{1}\right) \geqslant 0$. We now investigate the sign of $j$.

As $0<\frac{1}{\mu}<x_{1}<\frac{2}{\mu} \leqslant \frac{1}{2}$, it is sufficient to study $j$ on the interval $I=\left[0, \frac{1}{2}\right]$.

$j^{\prime}(x)=\mu(2 x-1)$, so $j$ is strictly decreasing on $I$. The discriminant of the quadratic equation $j(x)=0$ being $\mu(\mu-4) \geqslant 0$, the latter has two solutions $\frac{\mu \pm \sqrt{\mu(\mu-4)}}{2 \mu}$, which are equal when $\mu=4$ (i.e., when $a=\frac{1}{2}$ ). Note that only $\frac{\mu-\sqrt{\mu(\mu-4)}}{2 \mu}$ may belong to $I$, and that the latter is equal to $\frac{1}{2}-\sqrt{\frac{1}{4}-\frac{1}{\mu}}=\frac{1}{2}-\sqrt{\frac{1}{4}-a(1-a)}=\frac{1}{2}-\sqrt{\left(a-\frac{1}{2}\right)^{2}}$. We successively have

$$
\begin{aligned}
\frac{1}{2}-\sqrt{\left(a-\frac{1}{2}\right)^{2}} & =\frac{\frac{1}{4}-\left(a-\frac{1}{2}\right)^{2}}{\frac{1}{2}+\sqrt{\left(a-\frac{1}{2}\right)^{2}}} \\
& =\frac{-a^{2}+a}{\frac{1}{2}+\sqrt{\left(a-\frac{1}{2}\right)^{2}}} \\
& =\frac{-a(a-1)}{\frac{1}{2}+\sqrt{\left(a-\frac{1}{2}\right)^{2}}}
\end{aligned}
$$

which is positive for each $a \in] 0,1\left[\right.$ and less than $\frac{1}{2}$. Thus $\left.\frac{\mu-\sqrt{\mu(\mu-4)}}{2 \mu} \in\right] 0,1 / 2[$ and we have the variations depicted in Figure 7.

If $x_{1}$ belongs to $\left[0, \frac{\mu-\sqrt{\mu(\mu-4)}}{2 \mu}\right]$, then $j\left(x_{1}\right)$ is positive and $H\left(x_{1}\right)$ is negative. 
Let us show that $x_{1} \in\left[\frac{\mu-\sqrt{\mu(\mu-4)}}{2 \mu}, \frac{1}{2}\right]$. As $a \leqslant \frac{1}{\mathrm{e}^{2}+1}$, we then have $a-\frac{1}{2}<0$, and so $\sqrt{\left(a-\frac{1}{2}\right)^{2}}=\frac{1}{2}-a$. Finally, $\frac{\mu-\sqrt{\mu(\mu-4)}}{2 \mu}=a$. As stated at the beginning of the proof, since $x_{1}$ is a root of $H, x_{1}$ is a fixed point for $f_{a}$ and thanks to (7).

$a<x_{1}$, and so $j\left(x_{1}\right)<0$, as $j$ is decreasing with $j(a)=0$. To put it in a nutshell, $H\left(x_{1}\right)>0$.

\begin{tabular}{|c|lll|}
\hline$x$ & $-\infty$ & $+\infty$ \\
\hline$H^{\prime}(x)$ & & - & \\
\hline $\mathcal{C}_{H}$ & $+\infty$ & \\
& & $-\infty$ \\
\hline
\end{tabular}

Figure 5. Variations of $\mathrm{H}$.

\begin{tabular}{|c|cccccc|}
\hline$x$ & $-\infty$ & & $x_{1}$ & $\frac{2}{\mu}$ & $x_{2}$ & $+\infty$ \\
\hline$H^{\prime}(x)$ & & - & 0 & + & 0 & - \\
\hline $\mathcal{C}_{H}$ & $+\infty$ & & & \\
\end{tabular}

Figure 6. Variation of $\mathrm{H}$ when $a \leqslant \frac{1}{\mathrm{e}^{2}+1}$.

\begin{tabular}{|c|c|c|c|}
\hline$x$ & 0 & $\frac{\mu-\sqrt{\mu(\mu-4)}}{2 \mu}$ & $\frac{1}{2}$ \\
\hline$j^{\prime}(x)$ & - & & - \\
\hline$j(x)$ & + & 0 & - \\
\hline $\mathcal{C}_{j}$ & 1 & 0 & $\longrightarrow 1-$ \\
\hline
\end{tabular}

Figure 7. Variations of $j$.

A numerical simulation based on a dichotomic approach to solve the equation

$$
\bar{x}=\frac{1}{1+\lambda(a) \mathrm{e}^{-\mu(a) \bar{x}}}
$$

on $] 0,1[$, leads to the curve depicted in Figure 8. 


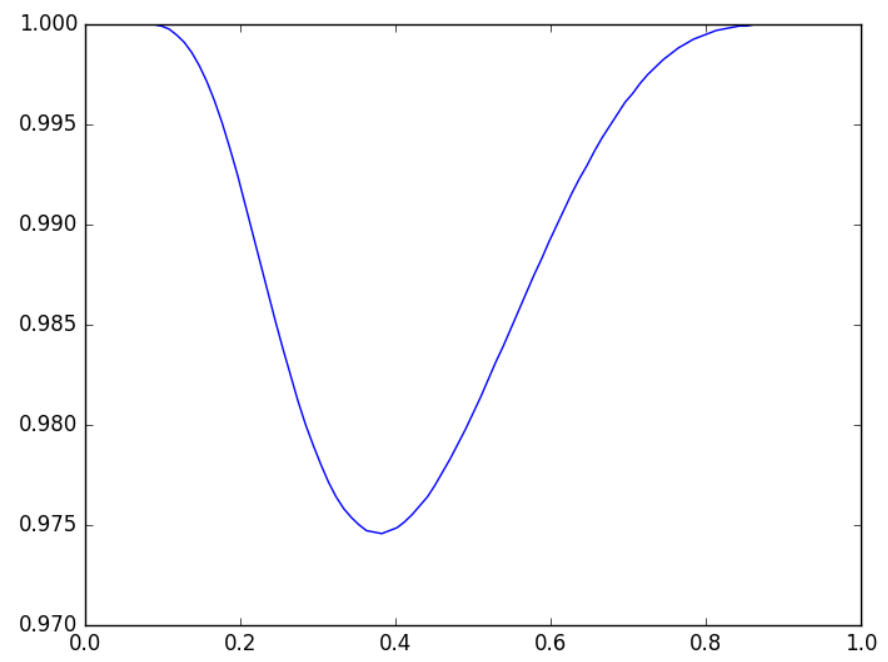

Figure 8. Graph of numerical solution of equation $\bar{x}=\frac{1}{1+\lambda(a) \mathrm{e}^{-\mu(a) \bar{x}}}$ when $a$ is varying in $] 0,1[$.

Let us now establish the following result:

Proposition 1. For all $x \in] 0,1\left[,\left|f_{a}^{\prime}(x)\right|<1\right.$.

Proof. As stated previously (Equation (3)), $f_{a}^{\prime}(x)=\mu f_{a}(x)\left(1-f_{a}(x)\right)$. So, we have

$$
\begin{aligned}
f_{a}^{\prime \prime}(x) & =\mu f_{a}^{\prime}(x)\left(1-f_{a}(x)\right)-\mu f_{a}(x) f_{a}^{\prime}(x) \\
& =\mu f_{a}^{\prime}(x)\left(1-2 f_{a}(x)\right) .
\end{aligned}
$$

Thanks to inequation (2) it can be deduced that $f_{a}^{\prime \prime}(x)$ shares then the same sign than $1-2 f_{a}$ on $\mathbb{R}$, and consequently on $[0,1]$ where the fixed point $\bar{x}$ is located. Furthermore,

$$
\begin{aligned}
1-2 f_{a}(x) \leqslant 0 & \Longleftrightarrow f_{a}(x) \geqslant \frac{1}{2} \Longleftrightarrow \frac{1}{1+\lambda e^{-\mu x}} \geqslant \frac{1}{2} \Longleftrightarrow 1+\lambda e^{-\mu x} \leqslant 2 \\
& \Longleftrightarrow e^{-\mu x} \leqslant \frac{1}{\lambda} \Longleftrightarrow x \geqslant \frac{\ln (\lambda)}{\mu} .
\end{aligned}
$$

But $\frac{\ln (\lambda)}{\mu} \geqslant 0 \Longleftrightarrow \lambda \geqslant 1 \Longleftrightarrow \frac{1}{a}-1 \geqslant 1 \Longleftrightarrow a \leqslant \frac{1}{2}$.

So, if $a \geqslant \frac{1}{2}$, then $\frac{\ln (\lambda)}{\mu} \leqslant 0$, and thus $\forall x \in[0,1], x \geqslant \frac{\ln (\lambda)}{\mu}$. This implies that $\forall x \in[0,1], 1-2 f_{a}(x) \leqslant 0$, and so $\forall x \in[0,1], f_{a}^{\prime \prime}(x) \leqslant 0$. In other words, if $a \geqslant \frac{1}{2}$, then $f_{a}^{\prime}$ decreases in $[0,1]$, and so:

$$
\forall x \in[0,1], 0 \leqslant f_{a}^{\prime}(x) \leqslant f_{a}^{\prime}(0)=a<1 .
$$

To sum up, if $a \geqslant \frac{1}{2}$, then $f_{a}$ is a contraction mapping such that $\left|f_{a}^{\prime}\right|$ is bounded by $a$.

Let us now consider $a<\frac{1}{2}$, and $\check{x}=\frac{\ln (\lambda)}{\mu}$. $\check{x}$ is in $[0,1]$, as

$$
\frac{\ln (\lambda)}{\mu}=\frac{\ln \left(\frac{1-a}{a}\right)}{\frac{1}{a(1-a)}} \leqslant \frac{\frac{1-a}{a}-1}{\frac{1}{a(1-a)}}=(1-2 a)(1-a),
$$


and each term of the right-side product is in $[0,1]$. Additionally, as $1-2 f_{a}(x) \leqslant 0 \Longleftrightarrow x \geqslant \frac{\ln (\lambda)}{\mu}=\check{x}$ and as $\check{x}<1$, we can deduce that the sigh of $f_{a}^{\prime \prime}$, which is the one of $1-2 f_{a}(x)$, is negative for $x \geqslant 1$. Finally, $\lim _{x \rightarrow+\infty} f_{a}^{\prime}(x)=\lim _{x \rightarrow+\infty} \frac{\lambda \mu e^{-\mu x}}{\left(1+\lambda e^{-\mu x}\right)^{2}}=0$. All these information are summarized in Figure 9.

$f_{a}^{\prime}(x)$ has a maximum in $\check{x}$, but $f_{a}^{\prime}(\check{x})=\frac{\mu}{4}<1$, since $\mu<4$. In fact the function $a(1-a)$ has a maximum in $a=1 / 2$. Since $f_{a}^{\prime}$ is also positive on $[0,1]$ we have proven that $\left|f_{a}^{\prime}(x)\right|<1$, for all $x \in[0,1]$.

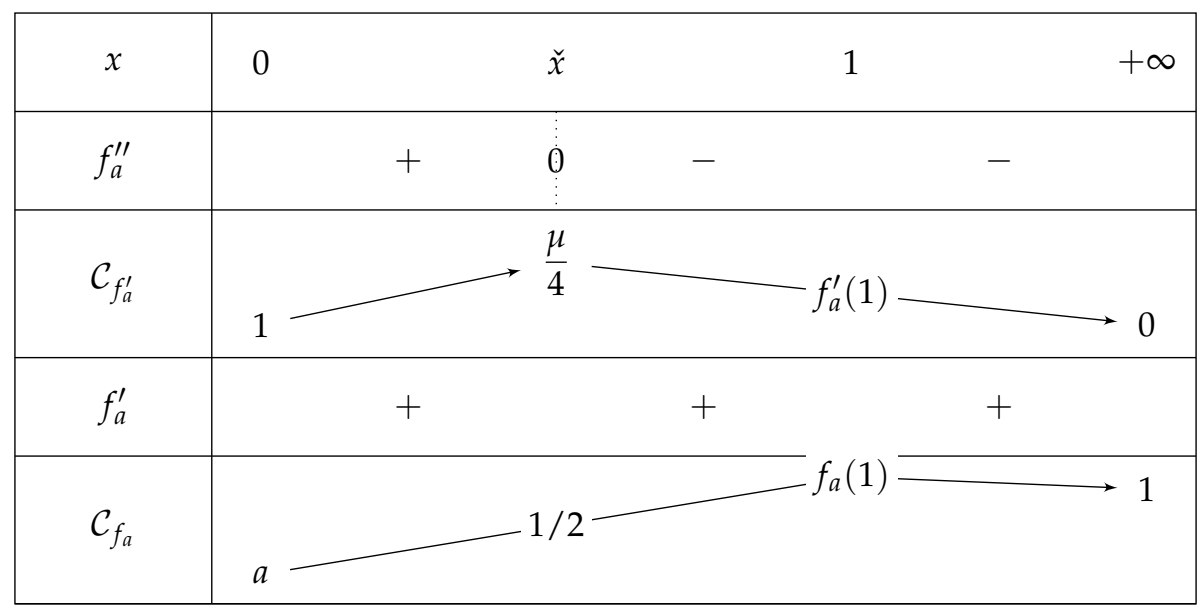

Figure 9. Behavior of $f_{a}$.

From all the material detailed previously, we can thus conclude that,

Theorem 2. The unique fixed-point of $f_{a}$ is an attractive one.

Proof. As $\left|f_{a}^{\prime}(x)\right|<1, f_{a}$ is a contraction mapping. By applying the Banach fixed-point theorem, we can deduce again the existence and uniqueness, and in addition the exponential convergence of the dynamical system to this fixed point.

Note that:

1. The unique fixed-point can be found as follows: start with an arbitrary element $x_{0}$ in $\mathbb{R}$ and define a sequence $\left(x_{n}\right)_{n \in \mathbb{N}}$ by $x_{n}=f_{a}\left(x_{n-1}\right)$, then $x_{n} \longrightarrow \bar{x}(a)$.

2. For $a \geqslant 1 / 2$, as $\left|f_{a}^{\prime}(x)\right|<a$, we can deduce that $f_{a}$ is Lipschitz continuous, with a Lipschitz constant equal to $a$. As a well-known consequence, the convergence of the aforementioned sequence is at least geometric, with a common ratio of $a$. For $a<1 / 2$ the same conclusion holds but for a constant $\gamma<1$.

\section{The 1-Dimensional Situation $(n=1)$}

\subsection{The Discrete Dynamical System under Consideration}

Let us firstly recall the general model to study gene networks. Let $n \in \mathbb{N}^{*}, M \in \mathcal{M}_{n}(\mathbb{R})$ be a square real matrix of size $n \times n$, and let $X_{0} \in \mathbb{R}^{n}$. We consider the discrete dynamical system:

$(\Sigma) \forall k \in \mathbb{N}, X_{k+1}=F_{a}\left(M X_{k}\right)$ 
where

$$
\begin{aligned}
F_{a}: \quad \mathbb{R}^{n} & \longrightarrow \mathbb{R}^{n} \\
\left(x_{1}, \ldots, x_{n}\right) & \longmapsto\left(f_{a}\left(x_{1}\right), \ldots, f_{a}\left(x_{n}\right)\right) .
\end{aligned}
$$

Note that, most of the times, $X_{0}=(a, \ldots, a)^{T}$. We will now focus on its most simplest cases.

\subsection{A Fundamental Case: $M=(1)$}

Let us consider first that $n=1$ and that the matrix $M$ is the identity: $M=(1)$. The $(\Sigma)$ system becomes:

$$
\left\{\begin{array}{l}
x_{0}=a \\
x_{k+1}=f_{a}\left(x_{k}\right) .
\end{array}\right.
$$

Let us recall that, if the recurrent sequence $u_{k+1}=f\left(u_{k}\right)$ converges, then the limit is a fixed point of $f$. And, from the study of the previous section concerning the sigmoid $f_{a}$, we know that this fixed-point $\bar{x}(a)$ :

1. exists and is unique,

2. is such that $0 \leqslant a<\bar{x}(a)<1$,

3. the convergence speed is geometric, of ratio equal to $a(\gamma)$.

As $f_{a}$ is increasing, then the sequence $\left(x_{k}\right)_{k \in \mathbb{N}}$ is monotonic. Being bounded, as $f_{a}$ outputs are in $] 0,1$, we can conclude that the sequence converges, and thus its limit is $\overline{x_{a}}$. Finally, if $x_{0}<\bar{x}(a)$, then the sequence $\left(x_{n}\right)_{n \in \mathbb{N}}$ increases to its limit, and otherwise it decreases to its limit $\bar{x}(a)$, as depicted in Figure 10.

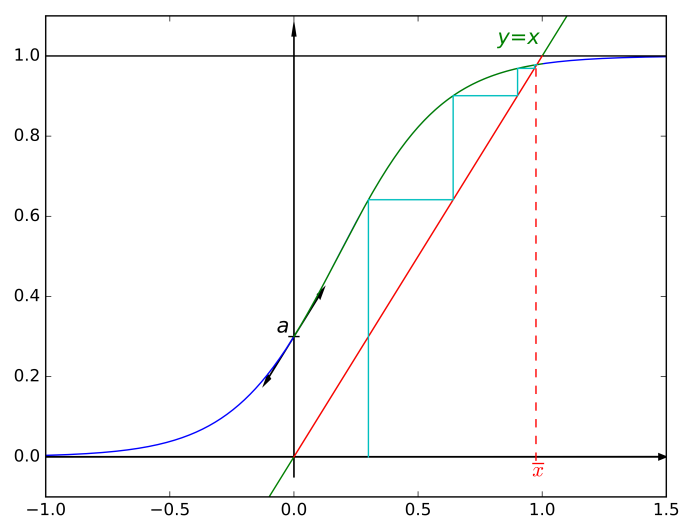

(a) $a=0.3, x_{0}=0.3$

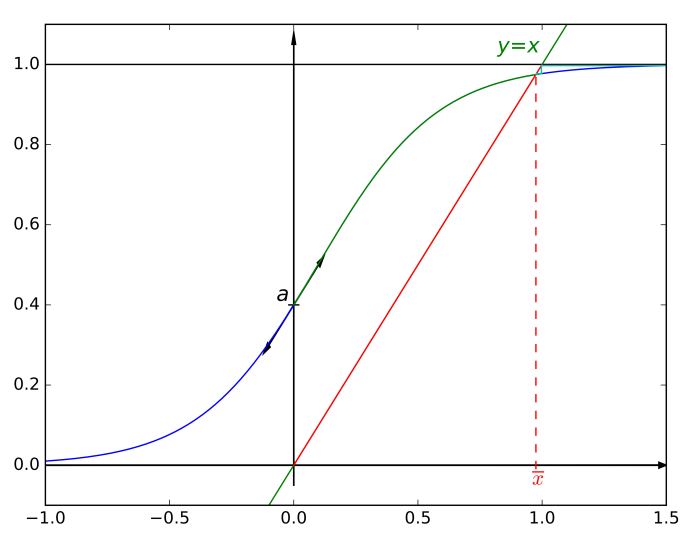

(b) $a=0.4, x_{0}=1.5$

Figure 10. Iterations of $x_{n+1}=f_{a}\left(x_{n}\right)$.

3.3. General 1-D Case: $M=(m)$

In that case, $(\Sigma)$ becomes:

$$
\left\{\begin{array}{l}
x_{0}=a \\
x_{k+1}=f_{a}\left(m x_{k}\right), \quad m \notin\{0,1\} .
\end{array}\right.
$$

Let us introduce $g_{a, m}(x)=f_{a}(m x)$. We investigate the fixed points of $g_{a, m}$ according to the sign of $m$.

3.3.1. Fixed Points of $g_{a, m}$ When $m>0$

First, $g_{a, m}(x)=x \Longleftrightarrow x=\frac{1}{1+\lambda \mathrm{e}^{-\mu m x}} \Longleftrightarrow \frac{1}{1+\lambda \mathrm{e}^{-\mu m x}}\left(1-x-\lambda x \mathrm{e}^{-\mu m x}\right)=0$. 
As in the fundamental case of Section 2.3, we are left to study the zeroes of function $H_{m}(x)=1-x-\lambda x \mathrm{e}^{-\mu m x}$, by investigating first its variations. We have $H_{m}^{\prime}(x)=\lambda \mathrm{e}^{-\mu m x}(\mu m x-1)-1$, and so $H_{m}^{\prime \prime}(x)=\lambda \mu m e^{-\mu m x}(2-\mu m x)$. The second order derivative $H_{m}^{\prime \prime}$ is positive if and only if (1) $m>0$ and $2-\mu m x>0$, or (2) $m<0$ and $2-\mu m x<0$. Both cases are equivalent to $x<\frac{2}{\mu m}$. We then have two variations for $H_{m}^{\prime}$, depending on the sign of $m$. First of all, Figure 11 depicts the case $m>0$.

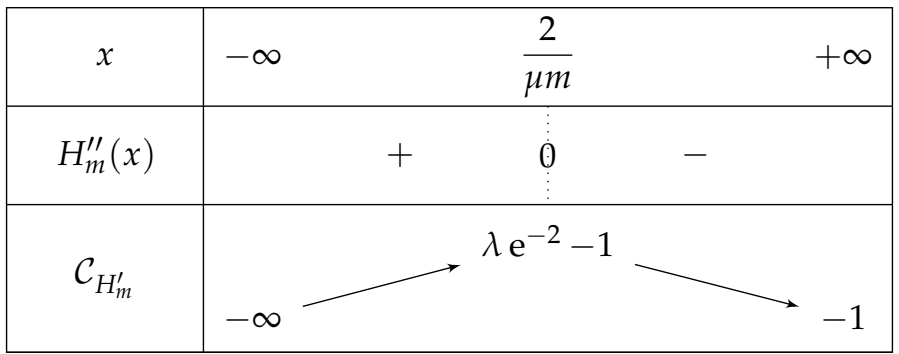

Figure 11. Variations of $H_{m}^{\prime}$ for $m>0$.

- If $a \geqslant \frac{1}{\mathrm{e}^{2}+1}$, then $\lambda e^{-2}-1$ is negative and then $H^{\prime} \leqslant 0$ over $\mathbb{R}$, and after the computation of the limits of $H_{m}$ as $x$ approaches $\pm \infty$, we can deduce the table of variations depicted in Figure 12 (and which is independent of $m$ ).

Consequently, if $a \geqslant \frac{1}{\mathrm{e}^{2}+1}$, then $H_{m}(x)=0$ has one unique solution, i.e., $g_{a, m}$ has one unique fixed point.

- If $a \leqslant \frac{1}{\mathrm{e}^{2}+1}$, then we obtain a curve similar to the fundamental case for $H_{m}$, see Figure 13 .

As previously, we remark that $H_{m}^{\prime}\left(x_{1}\right)=0=\lambda \mathrm{e}^{-\mu m x_{1}}\left(\mu m x_{1}-1\right)-1$, so $\lambda \mathrm{e}^{-\mu m x_{1}}=\frac{1}{\mu m x_{1}-1}$. As a consequence,

$$
\begin{aligned}
H_{m}\left(x_{1}\right) & =-\left(\frac{x_{1}}{\mu m x_{1}-1}+x_{1}-1\right) \\
& =\frac{-\left(\mu m x_{1}^{2}-\mu m x_{1}+1\right)}{\left(\mu m x_{1}-1\right)} .
\end{aligned}
$$

Again as previously, $H_{m}^{\prime}\left(\frac{1}{m \mu}\right)=-1<0, H_{m}^{\prime}$ is increasing over $\left.]-\infty, \frac{2}{\mu m}\right]$, and $H_{m}^{\prime}\left(x_{1}\right)=0$, so $x_{1}>\frac{1}{\mu m}$. Consequently, since $m>0$, then $\mu m x_{1}-1>0$, and $H_{m}\left(x_{1}\right)$ has the opposite sign of $j_{m}\left(x_{1}\right)$ where $j_{m}(x)=\mu m x^{2}-\mu m x+1$.

Let us study the quadratic polynomial $j_{m}(x)$ on $\mathbb{R}$. Its discriminant $\Delta\left(j_{m}\right)$ is equal to $\mu^{2} m^{2}-4 \mu m=\mu m(\mu m-4)$, and it has the sign table described in Figure 14.

- If $m \in] 0, \frac{4}{\mu}\left[\right.$, then $j_{m}\left(x_{1}\right)=\mu m x_{1}^{2}-\mu m x_{1}+1=\mu m\left(x_{1}-\frac{1}{2}\right)^{2}-\frac{\mu m}{4}+1$. As $0<m<\frac{4}{\mu}$, we can conclude that $j_{m}\left(x_{1}\right)>0$. So $H_{m}\left(x_{1}\right)<0$. For the same reasons, $H_{m}\left(x_{2}\right)$ is negative and $H_{m}$ has thus only one root, which belongs to $]-\infty, x_{1}\left[\right.$. Thus $g_{m, a}$ has only one fixed point in this interval.

- $\quad$ If $m=\frac{4}{\mu}$, then

$$
H_{\frac{4}{\mu}}\left(x_{1}\right)=\frac{-\left(2 x_{1}-1\right)^{2}}{\left(1+\lambda \mathrm{e}^{-4 x_{1}}\right)\left(4 x_{1}-1\right)},
$$


which is of the sign of $1-4 x_{1}$. But $x_{1}>\frac{1}{m \mu}=\frac{1}{4} \Longrightarrow 4 x_{1}>1 \Longrightarrow 1-4 x_{1}<0$. So, for $m=\frac{4}{\mu}, H_{\frac{4}{\mu}}\left(x_{1}\right)$ is strictly negative and $H_{\frac{4}{\mu}}\left(x_{2}\right)$ similarly. Again, $g_{\frac{4}{\mu}}, a$ has only one fixed point in the interval $]-\infty, x_{1}[$.

- If $m>\frac{4}{\mu}$, then $\Delta\left(j_{m}\right)>0$. So $j_{m}$ is positive outside its two roots and negative otherwise. $\frac{1}{2} \pm \sqrt{\frac{1}{4}-\frac{1}{m \mu}}$. The largest one is outside $I=\left[0, \frac{1}{2}\right]$. Let us first focus on $z_{1}=\frac{1}{2}-\sqrt{\frac{1}{4}-\frac{1}{m \mu}}=\frac{\frac{1}{\mu m}}{\frac{1}{2}+\sqrt{\frac{1}{4}-\frac{1}{\mu m}}}$. Since $0 \leqslant \frac{1}{\mu m} \leqslant \frac{1}{4}$ then $\frac{1}{2} \leqslant \frac{1}{2}+\sqrt{\frac{1}{4}-\frac{1}{\mu m}} \leqslant 1$ and thus $\frac{1}{\mu m} \leqslant z_{1} \leqslant \frac{2}{\mu m}$. From $\frac{1}{\mu m} \leqslant z_{1} \leqslant \frac{1}{2}$, one can thus deduce that $\frac{2}{\mu m} \leqslant z_{2} \leqslant 1$. Thus, $\frac{2}{\mu m}$ is in $] z_{1}, z_{2}\left[\right.$ and $j_{m}\left(\frac{2}{\mu m}\right)<0$. In other words,

$$
H_{m}\left(x_{2}\right)>H_{m}\left(\frac{2}{\mu m}\right)>0 .
$$

* If $\mu m$ is large (i.e., $\frac{1}{\mu m}$ is close to 0 ), $z_{1}$ is close to $\frac{1}{\mu m}$. The left root of $H, x_{1}$ would be s.t. $x_{1} \geq z_{1}$ and $j_{m}\left(x_{1}\right)<0$. In such a case $H_{m}\left(x_{1}\right)>0$ and there is only one fixed point in ]$x_{2}, \infty[$.

* If $\mu m$ is close to $4, z_{1}$ is close to $\frac{2}{\mu m}$. The left root of $H, x_{1}$ would be s.t. $x_{1} \leq z_{1}$ and $j_{m}\left(x_{1}\right)>0$. In such a case $H_{m}\left(x_{1}\right)<0$ and there is one fixed point in $]-\infty, x_{1}$ [, one in ]$x_{1}, \frac{2}{\mu m}[$ and one in $] x_{2}, \infty[$.

* if $x_{1}=z_{1}, j_{m}\left(x_{1}\right)=0$ and so $H_{m}\left(x_{1}\right)=0$. There is one fixed point $x_{1}$ in $\left.]-\infty, \frac{2}{\mu m}\right]$ and one in $] x_{2}, \infty[$.

\begin{tabular}{|c|c|c|}
\hline$x$ & $-\infty$ & $+\infty$ \\
\hline$H_{m}^{\prime}(x)$ & - & \\
\hline $\mathcal{C}_{H_{m}}$ & $+\infty$ & \\
\hline
\end{tabular}

Figure 12. Variation of $H_{m}$ for $a \geqslant \frac{1}{\mathrm{e}^{2}+1}$. 


\begin{tabular}{|c|cccccc|}
\hline$x$ & $-\infty$ & & $x_{1}$ & $\frac{2}{\mu m}$ & $x_{2}$ & $+\infty$ \\
\hline$H_{m}^{\prime}(x)$ & & - & 0 & + & $\vdots$ & 0 \\
$\vdots$ \\
\hline $\mathcal{C}_{H_{m}}$ & $+\infty$ & & \\
\end{tabular}

Figure 13. Variation of $H_{m}$ for $a \leqslant \frac{1}{\mathrm{e}^{2}+1}$.

\begin{tabular}{|c|c|c|c|c|c|}
\hline$m$ & & 0 & & $\frac{4}{\mu}$ & \\
\hline$\Delta\left(j_{m}\right)$ & + & Q & - & Q & + \\
\hline
\end{tabular}

Figure 14. Sign of $\Delta\left(j_{m}\right)$.

\subsubsection{Fixed Points of $g_{a, m}$ When $m<0$}

The existence of a unique fixed point can be proved without computations, however deeper investigation to establish the nature of this fixed pointed are necessary.

Lemma 1. $g_{a, m}(x)$ has at least one fixed point.

Proof. Suppose for an absurdum that $g_{a, m}$ has no fixed points. Since $g_{a, m}$ is a continuous map and $g_{a, m}(0)=a$ we have

$$
\Gamma\left(g_{a, m}\right):=\left\{(x, y): g_{a, m}(x)=y\right\} \subset\{(x, y): x<y\}
$$

that is trivially false since there are $x \in \mathbb{R}$ such that $x>g_{a, m}(x)$, (for instance $\left.x=2 / m\right)$.

Theorem 3. If $m<0$, then $g_{a, m}$ has a unique fixed point.

Proof. Let $P \subset \mathbb{R}^{2}$ be the subset of the fixed points of $f$, i.e., $P=\left\{(x, x) \in \mathbb{R}^{2}: f(x)=x\right\}$; since $P$ is a compact set, we can consider his minimum $p_{1} \in P$, that we may call the first fixed point of $f$. In fact $P=\Gamma(f) \cap \Gamma(y=x) \cap[0,1]^{2}$, thus $P$ is compact because is a finite intersection of close subsets contained in a compact set $\left([0,1]^{2}\right)$.

Suppose for an absurdum to have another fixed point $p_{2}$; since $p_{1}$ is the first fixed point, we have $p_{1}<p_{2}$. However $g_{a, m}$ is a decreasing function on $[0,1]$, in fact

$$
g_{a, m}^{\prime}(x)=\frac{\lambda \mu m \mathrm{e}^{-\mu m x}}{\left(1+\lambda \mathrm{e}^{-\mu m x}\right)^{2}},
$$

thus we have

$$
p_{1}=g_{a, m}\left(p_{1}\right)>g_{a, m}\left(p_{2}\right)=p_{2},
$$

a contradiction that proves that $p_{1}$ can be the only fixed point of $g_{a, m}$.

As can be seen, the number of fixed-point of $g_{a, m}$ can be deduced theoretically, according to the values of $a$ and $m$. At each fixed-point $\bar{x}$, we still have to study its attractive property thanks to the value of $\left|g_{a, m}^{\prime}(\bar{x})\right|$. Although technical, this latter can be done by using usual methods from the mathematical analysis. 


\section{Conclusions and Future Work}

In this article, the objective was to show that gene-network models like the Wagner one, based on the iterations of some discrete dynamical systems defined using a sigmoid function, can be studied too theoretically. Iterations of the sigmoid function has been deeply studied in a first section, emphasizes the fact that the number of fixed points, their approximate location, and their attractivity, can be computed mathematically. Furthermore, we shown that each iteration of this sigmoid function tends to the fixed-point, no matter the initial condition. Elements showing how to extend this study to the complete one self-regulating gene has then be provided in a second section, showing the possibility of such studies. In future work, we intend to extend such investigations to a network of more than one gene. We will first deeply studied the case of 2-4 genes. Such investigations will then be extended to a larger number of genes, introducing qualitative methods that will depend on the shape of the considered matrix.

Author Contributions: Christophe Guyeux outlined and structured the demonstration of the general case, in its first version, and all the proofs have been checked and improved by Jean-François Couchot. and then Luigi Marangio, who proposed the solution for the case $m<0$ in Section 3. Finally, Arnaud Le Rouzic proposed the framework and the related works, while Jacques M. Bahi supervised the work.

Conflicts of Interest: The authors declare no conflict of interest.

\section{References}

1. Bahi, J.M.; Guyeux, C.; Perasso, A. Chaos in DNA evolution. Int. J. Biomath. (IJB) 2016, 9, 1650076.

2. Guyeux, C.; Nicod, J.M.; Philippe, L.; Bahi, J.M. The study of unfoldable self-avoiding walks. Application to protein structure prediction software. JBCB J. Bioinform. Comput. Biol. 2015, 13, 1550009.

3. Pai, A.A.; Pritchard, J.K.; Gilad, Y. The genetic and mechanistic basis for variation in gene regulation. PLoS Genet. 2015, 11, e1004857.

4. Bornholdt, S. Modeling genetic networks and their evolution: A complex dynamical systems perspective. Biol. Chem. 2001, 382, 1289-1299.

5. Barabási, A.L.; Oltvai, Z.N. Network biology: Understanding the cell's functional organization. Nat. Rev. Genet. 2004, 5, 101-113.

6. Polynikis, A.; Hogan, S.; di Bernardo, M. Comparing different ODE modelling approaches for gene regulatory networks. J. Theor. Biol. 2009, 261, 511-530.

7. Perc, M. Stochastic resonance on paced genetic regulatory small-world networks: Effects of asymmetric potentials. Eur. Phys. J. B 2009, 69, 147-153.

8. Gosak, M.; Markovič, R.; Dolenšek, J.; Rupnik, M.S.; Marhl, M.; Stožer, A.; Perc, M. Network science of biological systems at different scales: A review. Phys. Life Rev. 2018, 24, 118-135.

9. Wagner, A. Does evolutionary plasticity evolve? Evolution 1996, 50, 1008-1023.

10. Fierst, J.L.; Phillips, P.C. Modeling the evolution of complex genetic systems: The gene network family tree. J. Exp. Zool. Part B Mol. Dev. Evol. 2015, 324, 1-12.

11. Wagner, A. Evolution of gene networks by gene duplications: A mathematical model and its implications on genome organization. Proc. Natl. Acad. Sci. USA 1994, 91, 4387-4391.

12. Masel, J. Genetic assimilation can occur in the absence of selection for the assimilating phenotype, suggesting a role for the canalization heuristic. J. Evolut. Biol. 2004, 17, 1106-1110.

13. Pinho, R.; Borenstein, E.; Feldman, M.W. Most networks in Wagner's model are cycling. PLoS ONE 2012, 7, e34285.

14. Siegal, M.L.; Bergman, A. Waddington's canalization revisited: Developmental stability and evolution. Proc. Natl. Acad. Sci. USA 2002, 99, 10528-10532.

15. Carneiro, M.O.; Taubes, C.H.; Hartl, D.L. Model transcriptional networks with continuously varying expression levels. BMC Evolut. Biol. 2011, 11, 363.

16. Rünneburger, E.; Le Rouzic, A. Why and how genetic canalization evolves in gene regulatory networks. BMC Evolut. Biol. 2016, 16, 239. 
17. Huerta-Sanchez, E.; Durrett, R. Wagner's canalization model. Theor. Popul. Biol. 2007, 71, 121-130.

18. Le Cunff, Y.; Pakdaman, K. Phenotype-genotype relation in Wagner's canalization model. J. Theor. Biol. 2012, 314, 69-83.

() 1

(C) 2018 by the authors. Licensee MDPI, Basel, Switzerland. This article is an open access article distributed under the terms and conditions of the Creative Commons Attribution (CC BY) license (http://creativecommons.org/licenses/by/4.0/). 